\title{
A CHARACTERIZATION OF THE 2-SPHERE IN TERMS OF JORDAN CURVE SEPARATION
}

\author{
CARSTEN THOMASSEN
}

(Communicated by James E. West)

\begin{abstract}
We show that, up to homeomorphism, the 2-sphere is the only compact arcwise connected metric space $X$ satisfying (i) If $J$ is a simple arc in $X$, then $X \backslash J$ is arcwise connected;

(ii) If $J$ is a simple closed curve in $X$, then $X \backslash J$ is disconnected (in the sense that $X \backslash J$ contains a proper nonempty subset that is both open and closed).
\end{abstract}

\section{INTRODUCTION}

Decades ago topologists found several results similar to that of the abstract. Early variants were found by Zippin [7] and extended by van Kampen [5]. Another related result is the "Kline Sphere Problem" [1], which, according to James E. West, was one of R. H. Bing's favourites of his own work. More recently, differential geometers seek characterizations of the spheres in terms of curvature and other metric invariants, see, e.g., [2]. In [1] and [5] local connectedness plays an important role. The purpose of this note is to prove a version that does not require any local topological property explicitly.

\section{Proof of the Characterization}

Zippin [7] proved that every compact, locally connected metric space satisfying (i) and (ii) of the abstract is homeomorphic to the 2-sphere. So, in order to prove the statement of the abstract it suffices to prove that the space $X$ in the abstract is locally connected. For this the following result of Moore [3] is useful.

Theorem 1. A compact, connected metric space $X$ is locally connected if and only if every pair of distinct points in $X$ can be separated in $X$ by a finite union of locally connected continua.

The space $X$ in the abstract satisfies Moore's criterion. In fact we have

Proposition 2. Let $X$ be an arcwise connected Hausdorff space satisfying (i) and (ii). Then any two points $p, q$ of $X$ can be separated by some simple

Received by the editors December 20, 1990.

1991 Mathematics Subject Classification. Primary 54E45. 
closed curve $J$ in the sense that $p$ and $q$ belong to distinct arcwise connected components of $X \backslash J$.

As pointed out by the referee, Proposition 2, was proved by Wilder [6, Chapter III, $\S \S 4$ and 5] assuming local connectedness, but only using the hypothesis of Proposition 2. Another proof can be obtained from the result in [4], which implies that the space $X$ in Proposition 2 does not contain the graph $K_{3,3}$, which may be thought of as a simple closed curve with three pairwise disjoint crossing "chords." For if an arcwise connected Hausdorff space $Y$ satisfying (i) contains two points that cannot be separated into distinct arcwise connected components by a simple closed curve, then it is not difficult to find a $K_{3,3}$ in $Y$.

The two points $p$ and $q$ in Proposition 2 belong to distinct arcwise connected components $X_{1}$ and $X_{2}$, say, of $X \backslash J$. But $X \backslash J$ has precisely two arcwise connected components (see [4]). As $X \backslash J$ is disconnected, by (ii), $X_{1}$ is open and closed in $X \backslash J$. Hence $p$ and $q$ are also separated in the sense required in Moore's criterion. So, Proposition 2 and Theorem 1 reduce the statement in the abstract to the result of Zippin.

\section{ACKNOWLEDGMENT}

Thanks are due to the referee for providing the reference [3] that substantially shortened the orginal proof.

\section{REFERENCES}

1. R. H. Bing, The Kline sphere characterization problem, Bull. Amer. Math. Soc. 52 (1946), 644-653.

2. K. Grove and K. Shiohama, A generalized sphere theorem, Ann. of Math. (2) 106 (1977), 201-211.

3. R. L. Moore, A characterization of a continuous curve, Fund. Math. 7 (1925), 302-307.

4. C. Thomassen, A link between the Jordan Curve Theorem and the Kuratowski Planarity Criterion, Amer. Math. Monthly 97 (1990), 216-218.

5. E. R. van Kampen, On some characterizations of 2-dimensional manifolds, Duke Math. J. 1 (1935), 74-93.

6. R. L. Wilder, Topology of manifolds, Amer. Math. Soc. Colloq., Publ. vol. XXXII, Amer. Math. Soc., Providence, RI, 1949.

7. L. Zippin, A characterization of the closed 2-cell, Amer. J. Math. 55 (1933), 207-217.

Mathematical Institute, Technical University of Denmark, Building 303 , DK-2800 Lyngby, Denmark 